\title{
RESPUESTA DEL MAÍZ AL NITRÓGENO Y LA ROTACIÓN CON CANAVALIA, BAJO DOS TIPOS DE LABRANZA. RÍO HATO, PANAMÁ, 1993-941
}

\author{
Román Gordón², Jorge Franco ${ }^{2}$, Nivaldo De Gracia², Andrés González ${ }^{2}$
}

\begin{abstract}
RESUMEN
Respuesta del maíz al nitrógeno y la rotación con Canavalia, bajo dos tipos de labranza. Río Hato, Panamá, 1993-94. Se realizó un experimento en dos años consecutivos para evaluar la respuesta del maíz a tres dosis de $\mathrm{N}(0,75$ y $150 \mathrm{~kg}$ de $\mathrm{N}$ en forma de urea/ha), bajo el sistema de siembra en rotación del maíz con la leguminosa Canavalia ensiformis, comparado con una siembra sin leguminosas. Las parcelas de rotación se establecieron al inicio de la época lluviosa (mayo). En septiembre de cada año se sembró un experimento con maíz. El mismo consistió en evaluar dos tipos de labranza. Se evalua-ron dos sistemas de labranza, incorporando la leguminosa y dejándola sobre la superficie del suelo. Además de evaluar el aporte de la parte aérea de las leguminosas, así como el efecto de sus componentes (forraje y raíces), se evaluó la dinámica del nitrógeno y cuantificó la equivalencia del $\mathrm{N}$ aportado por la canavalia. Se utilizó un diseño experimental de parcelas sub-divididas, las parcelas principales fueron los sistemas, arregla-dos en Bloques Completos al Azar, con tres repeticiones, en las sub-parcelas se evaluó la labranza y en las sub parcelas las dosis de N. Se encontró una respuesta'altamente significativa para: 1- el efecto residual de los sistemas de labranza, 2- la respuesta de la aplicación del N y 3-la interacción Dosis de N x Sistemas, resultó altamente significativa.
\end{abstract}

\begin{abstract}
The response ofmaize towards application ofnitrogen, in rotation with Canavalia, under two cultivation types, at Río Hato, Panamá, 1993-94. An experiment was carried out for two consecutive years in order to evaluate the response of maize with the legume Canavalia ensiformis, and compared to a crop without legumes. The rotative plots were established at the beginning of the rainy season (May). In September of each year we planted anotherexperiment with rice. We evaluated two types of cultivation: one consisting of the incorporation of present legumes and weeds, present in the field before showing, and the other one consisted of leaving them on the surface. We evaluated four systems: one plot where we cut the legumes and left the waste on the site $(\mathrm{CanR}+\mathrm{F})$; another plot where the legume grew, and then we cutthe vegetative section ofthe plant and extracted the waste out ofthe plot (CanR): another plot with no legume and where we dumped the waste from the preceding plot (CanF), and the last plot with no legumes at all (SLeg). We found a highly significant response to the residual effect of the systems, and the group with the highest yielding was the CanR and also the CanR+F, with 5.39 and 5.28 tJha, respectively, followed by the Sleg plot, with a yield of 2.50tJha. The response to $\mathrm{N}$ application was also very significant, wiht average yields of 2.82, 4.54 and $4.99 \mathrm{t} / \mathrm{ha}$, for levels of 0,75 and $150 \mathrm{~kg}$ of $\mathrm{N} / \mathrm{ha}$, respectively. The interaction of N Doses X Sistems was highly significant.
\end{abstract}

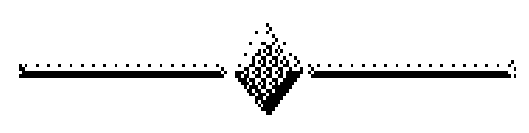

\section{INTRODUCCION}

El uso de leguminosas, como abonos verdes, es un método utilizado desde hace muchos años. Varios autores han informado de los beneficios de incorporar leguminosas de cobertura con el fin de incorporar nitrógeno (Wade y Sánchez, 1983; Yost, Evans, Saidy, 1985 y Barreto, et al., 1992). Bouldin,Quintana, Suheta, (1989) encontraron que se pueden sustituir hasta $170 \mathrm{~kg}$ de $\mathrm{N}$, con leguminosas de cobertura. Burle, et al. (1992), encontraron que la Canavalia brasiliensis y la Mucuna aterrima, son especies promisorias por su tolerancia a estrés hídrico y rápido crecimiento. Estos autores descubrieron que el maíz obtuvo hasta $80 \mathrm{~kg}$ de N/ha, provenientes de la canavalia. Durante 1992 Gordón, et al. (1994), evaluaron Mucuna deeringianum (mucuna) y Canavalia ensiformis (canavalia), sobresaliendo la canavalia. El rendimiento de maíz, en las parcelas en rotación con canavalia, superaron a las parcelas sin leguminosas, en 1,85 t/ha. El análisis económico de este ensayo determinó, que la dosis óptima de $\mathrm{N}$ para aplicar en este sistema fue de $54 \mathrm{~kg} / \mathrm{ha}$, mientras que, en las parcelas

\footnotetext{
1 Presentado en la XLII Reunión Anual del PCCMCA en El Salvador, Centroamérica, 1996

2 Instituto de Investigación Agropecuaria de Panamá (IDIAP), Centro Regional Agropecuario de Azuero, Panamá.
} 
que no tenían leguminosas en relevo, la dosis óptima fue de $139 \mathrm{~kg} / \mathrm{ha}$, produciendo un ahorro de $85 \mathrm{~kg}$ de N/ha. En 1993, Gordón (1994) realizó un experimento similar en donde evalúo el efecto de los componentes de las plantas (raíces y follaje) por separado, encontrando diferencias en la respuesta del maíz en cada sistema.

El presente trabajo se realizó con el objetivo de determinar los requerimientos de nitrógeno en forma de urea, para la producción del maíz en monocultivo, bajo un sistema de siembra alterno de canavalia en mayo y maíz en setiembre.

\section{MATERIALES Y MÉTODOS}

Se realizaron dos ensayos consecutivos (1993 y 1994), en la finca Río Hato Sur, Provincia de Coclé, República de Panamá, la cual está ubicada a unos 25 msnm. Se evaluó la respuesta del maíz a tres dosis de nitrógeno (0, 75 y $150 \mathrm{~kg} \mathrm{~N} / \mathrm{ha})$, bajo el sistema de siembra de rotación del maíz con la leguminosa Canavalia ensiformis, además de una siembra sin leguminosas. La leguminosa fue sembrada al inicio de la época lluviosa (mayo) y el maíz en setiembre del mismo año. Se evaluaron dos tipos de labranza, uno consistió en incorporar las leguminosas y malezas antes de la siembra (labranza convencional) y el otro en dejarlas sobre la superficie del suelo (labranza de conservación).

Para la realización de este ensayo se contó con cuatro parcelas, de un área de $324 \mathrm{~m}^{2}(27$ x $12 \mathrm{~m})$ :
1) Parcela donde creció la Canavalia, luego en septiembre se cortó el forraje y se dejó todo este material sobre la misma. Corresponde al efecto de ambos componentes de la planta (raíces y forraje) y del mejoramiento del suelo. Se le denominó $\mathrm{Ca}$ navalia Raíces + Forraje $(\mathrm{CanR}+\mathrm{F})$.

2) Parcela donde creció la Canavalia, luego se cortó toda la parte vegetativa de las plantas y se extrajo este forraje de la parcela, quedando únicamente en la misma, las raíces de las leguminosas y el efecto del mejoramiento del suelo. Corresponde al sistema denominado Canavalia-Raíces (CanR).

3) Parcela sin leguminosas, que en septiembre se le colocó todo el forraje de la Canavalia que había en la parcela mencionada en el punto 2. Esta fue considerada como el Sistema Canavalia-Forraje (CanF).

4) Una parcela sin leguminosas (SLeg).

El suelo donde se sembró el experimento es franco-arenoso con aproximadamente $5 \%$ de pendiente y con fertilidad de media a baja. Se realizó un análisis del suelo en mayo y otro antes de la siembra del maíz (setiembre) (Cuadro 1). En este ensayo se utilizó el híbrido de maíz P-8916.

El diseño experimental utilizado fue el de parcelas sub-divididas (Sistemas x Labranza x Dosis de N). Las parcelas principales fueron arregladas en bloques completos al azar con tres repeticiones. La parcela principal fue el Sistema. Las subparcelas fueron el tipo de labranza (convencional y conservación), mientras que las subparcelas fueron las dosis de nitrógeno.

Cuadro 1. Análisis de suelo, para cada parcela en rotación, Río Hato, Panamá, 1993-94.

\begin{tabular}{|c|c|c|c|c|c|c|c|c|c|c|}
\hline Sistema & $\mathbf{p H}$ & A-L-Arc & $\mathbf{P}$ & $\mathbf{K}$ & $\mathrm{Ca}$ & $\mathrm{Mg}$ & Al & M.O. & $\mathbf{F e}$ & $\mathbf{Z n}$ \\
\hline \multicolumn{11}{|c|}{ Canavalia } \\
\hline mayo 1993 & 5,6 & $66-18-18$ & 1,6 & 117,5 & 0,9 & 0,2 & 0,1 & 2,68 & 1,0 & 0,7 \\
\hline setiembre 1993 & 5,8 & $70-16-14$ & 2,6 & 208,8 & 0,9 & 0,2 & 0,1 & 2,01 & 1,5 & 0,5 \\
\hline mayo 1994 & 5,8 & $70-16-14$ & 2,6 & 210,8 & 0,8 & 0,2 & 0,1 & 2,35 & 1,5 & 0,5 \\
\hline setiembre 1994 & 5,6 & $66-14-20$ & 4,1 & 255,0 & 1,3 & 0,3 & 0,1 & 2,80 & 1,9 & 0,3 \\
\hline \multicolumn{11}{|c|}{ Sin Leguminosas } \\
\hline mayo 1993 & 5,6 & $58-20-22$ & 1,7 & 94,1 & 0,9 & 0,6 & 0,1 & 2,28 & 1,7 & 0,4 \\
\hline setiembre 1993 & 5,8 & $70-16-14$ & 1,9 & 180,0 & 0,6 & 0,2 & 0,1 & 2,55 & 1,1 & 0,2 \\
\hline mayo 1994 & 5,8 & $76-18-16$ & 1,5 & 47,0 & 0,6 & 0,2 & 0,1 & 1,74 & 1,0 & 1,3 \\
\hline setiembre 1994 & 5,8 & $62-18-20$ & 5,0 & 82,3 & 0,9 & 0,2 & 0,2 & 2,10 & 3,6 & 0,3 \\
\hline
\end{tabular}

$\mathrm{P}, \mathrm{K}, \mathrm{Fe}$ y $\mathrm{Zn}=(\mathrm{g} / \mathrm{ml}$

$\mathrm{Ka}, \mathrm{Mg}$ y $\mathrm{Al}=\mathrm{meq} / 100 \mathrm{ml}$

M.O. $=\%$ 
La parcela considerada como labranza convencional, consistió en una parcela en la que se procedió a dar tres pases de rastra liviana, incorporando todo el material vegetativo (forraje), que se encontraba en la superficie (leguminosas y malezas). La parcela de labranza de conservación, consistió en una parcela que no se preparó, sólo se chapeó la leguminosa y/o maleza (según el Sistema) y posteriormente se le aplicó el herbicida Glifosato a razón de 4 1/ha.

El tamaño de las unidades experimentales fue de cuatro surcos de maíz de 5,5 $\mathrm{m}$ de largo, separados a $0,75 \mathrm{~m}$ entre hileras y $0,50 \mathrm{~m}$ entre golpes, dejando dos plantas por golpe, para una densidad teórica de 5,33 plantas $/ \mathrm{m}^{2}$. El control de malezas después de la siembra del maíz, se realizó con la aplicación de la mezcla de atrazina más pendimentalina a razón de 2,0 + 2,0 1/ha, con posteriores limpias manuales, por escapes del control de algunas malezas. La fertilización consistió de la aplicación al momento de la siembra de $60 \mathrm{~kg}$ de $\mathrm{P}_{2} \mathrm{O}_{5}$ /ha más la mitad del nitrógeno. En 1994 se le aplicó, además del fósforo y el nitrógeno, la cantidad de 20 $\mathrm{kg}$ de $\mathrm{S}$ en forma de $\mathrm{Ca}_{2} \mathrm{SO}_{4}-2 \mathrm{H}_{2} \mathrm{O}$. El resto del nitrógeno se aplicó en forma de urea 30-35 días después de la siembra (dds). Se tomaron datos del peso y contenido de $\mathrm{N}$ de las leguminosas, al momento de cortarlas, contenido de nitrógeno en la hoja de la mazorca al momento de la floración, número de plantas y mazorcas a la cosecha, rendimiento y porcentaje de humedad del grano. La precipitación de esta localidad en los dos años de los experimento se observa en el Cuadro 2.

\section{RESULTADOS Y DISCUSIÓN}

Los resultados del análisis estadístico indicaron que hubo diferencias altamente significativas para las variables del rendimiento y algunos de sus componentes en los distintos factores estudiados, así como en algunas de las interacciones (Cuadro 3). En el análisis estadístico se observó que los factores Leguminosas, Dosis de $\mathrm{N}$ y Año absorbieron la mayor parte de la variación del experimento $(93,5 \%)$, con un $38,1,29,9$ y $25,5 \%$, respectivamente. Los otros componentes, a pesar de que ciertos presentan diferencias estadísticas, sólo alcanzaron el 7,5\% de la variación total del experimento para la variable rendimiento de grano. Los otros componentes del rendimiento o variables de respuestas siguieron la misma tendencia que la observada en el rendimiento.

\section{Producto de canavalia}

El porcentaje promedio de nitrógeno en tallos más hojas de canavalia fue de $3,20 \%$. La cantidad de mate-
Cuadro 2. Precipitación pluvial (mm) en el campo Experimental de Río Hato, Panamá, desde mayo hasta diciembre de 1993-94.

\begin{tabular}{lrrr}
\hline & \multicolumn{3}{c}{ Días } \\
Mes & $\mathbf{1 - 1 0}$ & $\mathbf{1 1 - 2 0}$ & $\mathbf{2 1 - 3 0 / 3 1}$ \\
\hline \multicolumn{3}{c}{1993} \\
may & 52,7 & 42,5 & 35,0 \\
jun & 0,0 & 7,5 & 100,0 \\
Jul & 59,9 & 31,0 & 31,4 \\
ago & 23,2 & 37,5 & 104,3 \\
sep & 22,4 & 12,6 & 59,9 \\
oct & 0,0 & 62,0 & 4,6 \\
nov & 32,5 & 142,8 & 83,5 \\
dic & 0,0 & 25,1 & 0,0 \\
\hline
\end{tabular}

1994

\begin{tabular}{lrrr} 
may & 55,0 & 97,9 & 103,5 \\
jun & 17,0 & 40,7 & 81,0 \\
jul & 40,7 & 11,8 & 4,9 \\
ago & 109,7 & 6,3 & 70,8 \\
set & 4,5 & 21,5 & 68,5 \\
oct & 128,7 & 45,9 & 80,2 \\
nov & 74,4 & 67,5 & 29,3 \\
dic & 29,0 & 0,0 & 0,0 \\
\hline
\end{tabular}

ria seca en las parcelas de canavalia, al momento de realizar el corte fue de 5,88 y 6,02 t/ha, para 1993 y 1994, respectivamente. Esto equivale a incorporar 188 y $192 \mathrm{~kg}$ de N/ha, en agosto de ambos años (Cuadro 4). La diferencia en el rendimiento de materia seca del forraje entre los dos años, se debió principalmente a la distribución de las lluvias durante el período de crecimiento de la leguminosa.

\section{Efecto residual de la canavalia}

Hubo una respuesta altamente significativa por el efecto residual de la canavalia y los distintos sistemas evaluados. Los rendimientos más altos se obtuvieron en las parcelas que tenían la canavalia y por último la parcela sin rotación con leguminosas. Esta respuesta índica que la siembra de la canavalia favoreció al cultivo.

Con relación a los sistemas, se encontró que el grupo de más alto rendimiento estuvo formado por CanR y CanR+F con 5,39 y 5,28 t/ha, respectivamente (no hubo diferencias estadísticas entre ellos). Luego le siguió CanF $(3,58)$ y por último la parcela SLeg con un rendimiento de2,50 t/ha. Este mismo orden se observó en los componentes peso de la mazorca y mazorcas por planta, 
Cuadro 3. Análisis de varianza de las variables rendimiento de grano y sus componentes, Río Hato, Panamá, $1993-94$.

\begin{tabular}{|c|c|c|c|c|c|c|}
\hline \multirow[b]{2}{*}{ F de Variación } & \multicolumn{6}{|c|}{ Cuadrados Medios } \\
\hline & g.l. & Rend & $\mathbf{P m z}$ & $\mathbf{P t m}^{2}$ & $\mathrm{Mzm}^{2}$ & Mz/pta \\
\hline Año & 1 & $42,85 * * 2$ & $9728 * *$ & $1,89 * *$ & $10,94 * *$ & $0,18 * *$ \\
\hline Rep(Año) & 2 & 0,30 & 102 & 0,01 & 0,04 & 0,002 \\
\hline Leg & 3 & $63,91 * *$ & $19587 * *$ & $0,42 * *$ & $7,83 * *$ & $0,29 * *$ \\
\hline Rep x Leg & 6 & 0,30 & 63,5 & 0,07 & 0,13 & 0,003 \\
\hline Lab & 1 & $0,28^{\text {n.s. }}$ & $2,81^{\text {n.s. }}$ & $0,03^{\text {n.s. }}$ & $0,39^{\text {n.s. }}$ & $0,02^{\text {n.s. }}$ \\
\hline Leg x Lab & 3 & $0,47^{\text {n.s. }}$ & $78,6^{\text {n.s. }}$ & $0,01^{\text {n.s. }}$ & $0,11^{\text {n.s. }}$ & $0,003^{\text {n.s. }}$ \\
\hline RepxLab(Leg) & 8 & 1,15 & 231,1 & $0,04^{\text {n.s. }}$ & 0,21 & 0,01 \\
\hline Nit & 2 & $50,32 * *$ & $11674 * *$ & $0,73^{\text {n.s. }}$ & $13,41 * *$ & $0,34 * *$ \\
\hline Leg x Nit & 6 & $2,22 * *$ & $877 * *$ & 0,10 & $1,74 * *$ & $0,07 * *$ \\
\hline Lab x Nit & 2 & $1,24 * *$ & $244^{*}$ & $0,13 * *$ & 0,14 n.s. & $0,01^{\text {n.s. }}$ \\
\hline LegxLabxNit & 6 & $0,31^{\text {n.s. }}$ & $100,8^{\text {n.s. }}$ & $0,11^{\text {n.s. }}$ & $0,28 *$ & $0,01^{\text {n.s. }}$ \\
\hline LegxAño & 3 & $2,13 * *$ & $1077^{* *}$ & 0,13 n.s. & $2,58 * *$ & $0,11 * *$ \\
\hline LabxAño & 1 & $0,03^{\text {n.s. }}$ & $7,8^{\text {n.s. }}$ & $0,005^{\text {n.s. }}$ & $0,12^{\text {n.s. }}$ & $0,001^{\text {n.s. }}$ \\
\hline NitxAño & 2 & $0,02^{\text {n.s. }}$ & $197^{*}$ & $0,06^{\text {n.s. }}$ & $3,81 * *$ & $0,15^{* *}$ \\
\hline LegxLabxAño & 3 & $1,61 * *$ & $514 * *$ & 0.09 n.s. & $0,26^{\text {n.s. }}$ & $0,01^{\text {n.s. }}$ \\
\hline LegxNitxAño & 6 & $0,17^{\text {n.s. }}$ & $119^{\text {n.s. }}$ & $0,10^{\text {n.s. }}$ & $0,67 * *$ & $0,02 * *$ \\
\hline LabxNitxAño & 2 & $0,22^{\text {n.s. }}$ & $235^{*}$ & $0,0002^{\text {n.s. }}$ & $0,15^{\text {n.s. }}$ & $0,004^{\text {n.s. }}$ \\
\hline Error & 80 & 0,204 & 70,4 & 0,069 & 0,131 & 0,004 \\
\hline C.V. $(\%)$ & & 10,86 & 10,15 & 5,18 & 7,53 & 7,25 \\
\hline
\end{tabular}

$\mathrm{Pmz}=$ Peso de mazorcas $\quad \mathrm{Ptm}^{2}$ y $\mathrm{Mzm}^{2}=$ plantas y mazorcas $/ \mathrm{m}^{2} \quad \mathrm{Mz} / \mathrm{Pt}=$ Mazorcas/planta

$*, * *, * * *$ se refieren a $\mathrm{P}<\mathrm{F}$ de $10 ; 5 ; 1$ y $0 ; 1 \%$, respectivamente. ${ }^{\text {n.s. }}$. No significativa estadísticamente.

Cuadro 4. Producción de materia seca de canavalia, Río Hato, Panamá, 1993-94.

\begin{tabular}{llccc}
\hline Canavalia & \multicolumn{1}{l}{ Rend t/ha } & \%N & kg N/ha \\
\hline Mat. verde & 5,88 & 1993 & 3,2 & 188,1 \\
\hline & & 1994 & \\
Mat. verde & 6,02 & & 3,2 & 192,6 \\
\hline
\end{tabular}

siendo sus diferencias significativas al 1 y $5 \%$, respectivamente. Esto indica que la siembra de las leguminosas antes del maíz, favoreció el rendimiento y los otros componentes de rendimiento del cultivo.

El número de plantas $/ \mathrm{m}^{2}$ presentó diferencias significativas a12\%, en donde el promedio de la población de los diferentes sistemas estuvo entre 5,19 a 4,94 plantas $/ \mathrm{m}^{2}$. El número de mazorcas $/ \mathrm{m}^{2}$ presentó diferencias al $1 \%$ de probabilidad, observándose el promedio más bajo en el sistema SLeg con 4,16 mazorcas $/ \mathrm{m}^{2}$. Con relación al tamaño de la mazorca, las más grande se lo- graron con los sistemas CanR y CanR+F con 105 y 100 $\mathrm{g} /$ mazorca, mientras que los tamaños más pequeños se obtuvieron con los sistemas CanF y SLeg (Cuadro 5).

Los resultados muestran que el sistema que sólo contenía las raíces (CanR), logró rendimientos superiores en comparación al sistema que evaluaba el efecto del forraje (CanF). Esto sugiere que la mayoóa del $\mathrm{N}$ que acumulan las hojas y tallos de las leguminosas, no es aprovechado por el cultivo en rotación. Las ganancias o respuestas positivas observadas en las parcelas

Cuadro 5. Rendimiento de grano y sus componentes, según sistemas evaluados, Río Hato, Panamá, 1993-94.

\begin{tabular}{lccccc}
\hline Sist & Rend. t/ha & Ptm $^{2}$ & Mzm $^{2}$ & Pmz g & MzPt \\
\hline CanR & 5,39 & 5,16 & 5,08 & 105 & 0,98 \\
CanR+F & 5,28 & 5,19 & 5,28 & 100 & 1,01 \\
CanF & 3,58 & 4.94 & 4,74 & 74 & 0,96 \\
SLeg & 2,50 & 5,10 & 4,16 & 53 & 0,81 \\
\hline
\end{tabular}


que sólo contenían las raíces, por su parte, sugieren que el mejoramiento físico que puede sufrir el suelo, además de la cantidad de $\mathrm{N}$ que es fijado durante el crecimiento o desarrollo de las leguminosas, así como, el contenido de $\mathrm{N}$ que tienen las raíces, son aprovechados o revierten en mayores beneficios para el cultivo de rotación. Además, debido al crecimiento de las raíces de la leguminosa, se pudo aumentar el contenido de micorrizas en el desarrollo del cultivo en rotación.

La respuesta de la aplicación del nitrógeno fue altamente significativa. Los rendimientos obtenidos para el promedio de las dosis de 0,75 y $150 \mathrm{~kg}$ de N/ha fue de 2,82, 4,54 y 4,99 t/ha, respectivamente. Con relación al peso de las mazorcas se observó que a medida que se aumenta la cantidad de $\mathrm{N}$, el tamaño de la mazorca fué mayor. Para las variables plantas $/ \mathrm{m}^{2}$ y mazorcas $/ \mathrm{m}^{2} \mathrm{se}$ observó que los promedios más bajos se encuentran en las parcelas sin nitrógeno (Cuadro 6).

Cuadro 6. Rendimiento de grano y sus componentes, según dosis de $\mathrm{N}$ aplicadas, Río Hato, Panamá, 1993-94.

\begin{tabular}{lrrr}
\hline Dosis de $\mathbf{N}$ & $\mathbf{0}$ & \multicolumn{1}{c}{$\mathbf{7 5}$} & $\mathbf{1 5 0}$ \\
\hline Rend (t/ha) & 2,82 & 4,54 & 4,99 \\
Pmz (g) & 61,87 & 89,09 & 95,12 \\
$\mathrm{Mz} / \mathrm{Pt}$ & 0,83 & 0,98 & 1,00 \\
$\mathrm{Ptm}^{2}$ & 4,93 & 5,13 & 5,20 \\
$\mathrm{Mzm}^{2}$ & 4,12 & 5,02 & 5,21 \\
\hline
\end{tabular}

\section{Efecto de la labranza}

En relación con el sistema de labranza, el mismo no mostró diferencias estadísticas, aunque, el promedio de las parcelas en labranza de conservación fue mayor, que las parcelas en labranza convencional ( 4,2 y 4,1 t/ha, respectivamente). La precipitación pluvial errática que se presenta comúnmente en esta zona del país, parece favorecer a las parcelas con residuos sobre la superficie, de tal manera que la poca humedad existente se conserva mejor.

Los otros componentes de rendimiento no mostraron diferencias significativas entre sí (Cuadro 7). Resultados similares encontró Gordón et al. (1994), de manera que el efecto de disminuir las pérdidas de la h!lmedad del suelo, sumado al efecto positivo encontrado en los sistemas que sólo incluyen las raíces, indican que es mejor dejar las plantas de la leguminosa a manera de mantillo y practicar la labranza de conservación. Contrario a estos resultados, Costa, Bouldin, Suhet (1990), encontraron que las parcelas donde no se incorporaban
Cuadro 7. Rendimiento y sus componentes, según tipo de labranza, Río Hato, Panamá, 1993-94.

\begin{tabular}{lrc}
\hline Labranza & Convencional & Conservación \\
\hline Rend & 4,11 & 4,20 \\
Pmz & 82,31 & 82,88 \\
$\mathrm{Mz} / \mathrm{Pt}$ & 0,93 & 0,95 \\
$\mathrm{Mzm}^{2}$ & 4,75 & 4,85 \\
$\mathrm{Ptm}^{2}$ & 5,08 & 5,11 \\
\hline
\end{tabular}

las leguminosas rendían menos, en comparación con las que sí hubo incorporación, debido a que en las no incorporadas había mayores pérdidas de nitrógeno.

\section{Respuesta de los sistemas a las dosis de nitrógeno}

La respuesta de la aplicación del $\mathrm{N}$ fue altamente significativa y dependió del manejo de los residuos de las leguminosas. En el análisis de varianza se observó que la interacción leguminosas por dosis de $\mathrm{N}$ fue significativa $(\mathrm{P}<0,01)$. Además del análisis de varianza, se realizó un análisis para determinar la curva de rendimiento máximo estable (plateau) en función de las dosis de $\mathrm{N}$ aplicado en forma de urca, para cada sistema (Cuadro 8).

El aporte de $\mathrm{N}$ al cultivo, por parte de los sistemas evaluados, se puede observar en el rendimiento del maíz en el intercepto de la curva con el eje del rendimiento, el cual representa el rendimiento del cultivo cuando no se aplica $\mathrm{N}$ al sistema. En el sistema SLeg, el valor del intercepto fue de $0,81 \mathrm{t} / \mathrm{ha}$. Este bajo rendimiento se puede explicar, por el bajo contenido de materia orgánica que muestran los distintos análisis del suelo en esta parcela. El incremento en el rendimiento de grano sin aplicar $\mathrm{N}$ a los sistemas CanR+F y CanR, fue significativamente superior, ya que, el valor del intercepto fue de 4,72 y 4,24 t/ha. Estos valores superan al testigo SLeg por el orden de 3,91 y 3,43 t/ha, respectivamente (Cuadro 8).

En este análisis se encontró que el rendimiento máximo (plateau) que se puede obtener en el sistema SLeg es de 3,86 t/ha, el cual es superado por los sistemas CanR, CanR+F en más de 1,5 t/ha, ya que, el plateau en estos fue de 5,91 y 5,50 t/ha, para cada sistema respectivamente. Este incremento en el techo del rendimiento, que se puede lograr implementando la rotación de la Canavalia en la producción del maíz, mejoraría la eficiencia de los productores en un 43 a $54 \%$.

El punto de inflexión de las curvas, indica la dosis en la cual la respuesta del cultivo permanece estable, es 
Cuadro 8. Valores para la curva de respuesta del rendimiento de grano, en función de las dosis de $\mathrm{N}$ aplicada, Río Hato, Panamá, 1993-94.

\begin{tabular}{lccccc}
\hline Sistemas & $\begin{array}{c}\text { Intercepto } \\
\left(\mathbf{b}_{\mathbf{0}}\right)\end{array}$ & $\begin{array}{c}\text { Pendiente } \\
\left(\mathbf{b}_{\mathbf{1}}\right)\end{array}$ & $\begin{array}{c}\text { RendMáximo } \\
(\text { Plateau })\end{array}$ & $\begin{array}{c}\text { Dosis N } \\
\text { de inflexión }\end{array}$ & $\mathbf{R}^{\mathbf{2}}$ \\
\hline Can R+F & 4,72 & 0,020 & 5,50 & 39 & $0,71^{*}$ \\
Can R & 4,24 & 0,055 & 5,91 & 30 & $0,97^{* *}$ \\
Can F & 2,14 & 0,060 & 4,30 & 36 & $0,94^{* *}$ \\
S Leg & 0,81 & 0,027 & 3,83 & 110 & $0,94^{* *}$ \\
\hline
\end{tabular}

$*, * *$ se refieren a $\mathrm{P}>\mathrm{F}$ de 1 y $0,1 \%$, respectivamente.

decir, el rendimiento no aumenta por incrementos en las dosis de $\mathrm{N}$ aplicado. $\mathrm{Al}$ analizar los valores encontrados se observa como en el sistema SLeg se necesitan 110 $\mathrm{kg} / \mathrm{ha}$, para lograr el rendimiento máximo, mientras que en los sistemas que involucran la canavalia, la dosis óptima varió entre 30 a $40 \mathrm{~kg} \mathrm{~N} / \mathrm{ha}$. Esta reducción implica un ahorro significativo en la utilización de urea en la producción del cultivo.

Se debe señalar que el efecto de la rotación de las leguminosas, parece involucrar algo más que el aporte de $\mathrm{N}$ calculado en base a la producción de biomasa, como lo puede ser, el mejoramiento en la estructura del suelo o algunos componentes que no se pudieron medir en este trabajo.

\section{Interacción sistemas por dosis de nitrógeno}

La dosis de cero nitrógeno, fue el factor que más reducción produjo en el número de mazorcas por planta, en las parcelas SLeg. Alrededor de un $42 \%$ de plantas abortaron las mazorcas en el nivel de $0 \mathrm{~N}$, en comparación con los niveles de 75 y $150 \mathrm{~kg}$ con 13 y $4 \%$ de aborto, respectivamente. En los sistemas CanR+F y CanR no se observó este efecto, ya que el porcentaje de aborto fue menor del 5\%, para todos los niveles. En el sistema CanF, el nivel de $0 \mathrm{~N}$ presentó $14 \%$ de plantas sin mazorcas. Los factores, plantas $/ \mathrm{m}^{2}$, mazorcas $/ \mathrm{m}^{2}$, peso de mazorca y número de mazorcas/planta, explican el porqué se obtuvieron bajos rendimientos con la dosis de cero N (Cuadro 9).

Cuando se observa el promedio general (a través de dosis) de los distintos sistemas, los datos sugieren que el forraje de la canavalia no tuvo mucho aporte en el rendimiento, ya que, no hubo diferencias significativas entre los sistemas CanR y $\mathrm{CanR}+\mathrm{F}$ (6,01 vs $6,00 \mathrm{y}$ 4,73 vs 4,59 t/ha, para los años 1993 y 1994, respectivamente).
Cuadro 9. Medias de rendimiento de grano y mazorcas por planta, obtenidos en el ensayo de rotación, según sistemas y dosis de N, Río Hato, Panamá, 1993-94.

\begin{tabular}{lccc}
\hline \multirow{2}{*}{ Sistemas } & \multicolumn{3}{c}{ Dosis de N } \\
\cline { 2 - 4 } & $\mathbf{0}$ & $\mathbf{7 5}$ & $\mathbf{1 5 0}$ \\
\hline \multirow{3}{*}{ Rend (t/ha) } \\
Can R+F & 4,68 & 5,43 & 5,56 \\
Can R & 4,25 & 5,86 & 5,96 \\
Can F & 2,14 & 4,02 & 4,56 \\
S Leg & 0,81 & 2,87 & 3,83 \\
\hline \multicolumn{5}{c}{ Maz/planta } \\
Can R+F & 0,99 & 1,04 & 1,01 \\
Can R & 0,93 & 1,01 & 1,01 \\
Can F & 0,87 & 0,98 & 1,03 \\
S Leg & 0,58 & 0,88 & 0,96 \\
\hline
\end{tabular}

Cuadro 10. Medias de rendimiento de grano obtenidos en el ensayo de rotación, según sistemas, dosis de $\mathrm{Ny}$ año, Río Hato, Panamá, 1993-94.

\begin{tabular}{lcccc}
\hline Sistemas & $\mathbf{0}$ & $\mathbf{7 5}$ & $\mathbf{1 5 0}$ & Prom \\
\hline & & 1993 & & \\
CanR & 4,77 & 6,56 & 6,72 & 6,01 \\
CanR+F & 5,60 & 6,10 & 6,17 & 6,00 \\
CanF & 2,41 & 4,07 & 4,85 & 3,78 \\
\hline & & 1994 & & \\
& & & & \\
CanR & 3,61 & 5,19 & 5,19 & 4,73 \\
CanR+F & 3,94 & 4,76 & 4,96 & 4,59 \\
CanF & 1,87 & 3,96 & 4,33 & 3,39
\end{tabular}


Al analizar la interacción LegxDosis en ambos años, se observó que al pasar de la dosis de 0 a $75 \mathrm{~kg}$ $\mathrm{N} /$ ha en el sistema CanR, hubo una ganancia de $1,76 \mathrm{y}$ $1,58 \mathrm{t} / \mathrm{ha}$, que representaron un 36,9 y $43,8 \%$ de incremento, con respecto a la dosis de $0 \mathrm{~N}$. Por el contrario, en el sistema CanR+F, el incremento en la dosis sólo aumentó los rendimientos en 0,50 y 0,82 t/ha $(8,9$ y $20,8 \%$ de incremento). En ambos años el nivel de ON en el sistema $\mathrm{CanR}+\mathrm{F}$ superó al rendimiento observado en el sistema de CanR. En el sistema CanF, este incremento de dosis representó un porcentaje mayor del rendimiento $(68,9$ y $111,7 \%)$. En los tres sistemas que involucraron la rotación con canavalia, se observó que no hubo respuesta a la aplicación de nitrógeno, cuando se pasó de la dosis de 75 a $150 \mathrm{~kg}$ Nlha (Cuadro 10).

\section{LITERATURA CITADA}

BARRETO, H.J; PÉREZ, C; FUENTES, M.R; QUEMÉ, J.L. 1992. Efecto de dosis de urea-N, insecticida y genotipo en el comportamiento del maíz (Zea mays L.) bajo un sistema de labranza mínima en rotación con dos leguminosas de cobertura. In: Síntesis de los Resultados Experimentales del PRM, 1991. pp 175-192.

BOULDIN,D.R;QUINTANA,J;SUHET,A. 1989. Evaluation potential of legume residues. In: (Claude, N. ed) Trop
Soils Technical Report. 1986-1987. North Caroline State University. Raleigh, N.C. pp 304-305.

BURLE, M; SUHET, A; PEREIRA, J; RESCK, D; PERES, J; CROAVO, M; BOWEN, W; BOULDIN, D; LATHWELL, D. 1992. Legume green manures: Dry season survi val and the effect on succeeding maize crops. Soil Management CRSP. NCSU, Raleigh N.C. Bulletin $\mathrm{N}^{\circ}$ 92, $35 \mathrm{p}$.

COSTA, F.S.A; BOULDIN, D.R; SUHET, A.R.1990. Evaluation of $\mathrm{N}$ recovery from mucuna placed on the surface or incorporated in a Brazilian Oxisol. Plant and SoiII24:91-96.

GORDON, R; FRANCO, J; DE GRACIA, N; MARTINEZ, L; GONZALEZ, A; DE HERRERA, A; BOLAÑOS, J. 1993. Respuesta del maíz a la aplicación de diferentes dosis de nitrógeno en rotación con canavalia y mucuna, bajo dos tipos de labranza, Río Hato, Panamá, 19921993. In: Síntesis de Resultados Experimentales del PRM, Vol. 4 p. 106-110.

WADE, M.K; SANCHEZ, P.A. 1983. Mulching and green manure applications for continuous crop production in the amazon basin. Agronomy Joumal. 75: 39-45.

YOST,R.S;EVANS,D.O; SAIDY,N.A. 1985. Tropical legumes for $\mathrm{N}$ production: growth and $\mathrm{N}$ content in relation to soil pH. Trop. Agric. (Trinidad). 62: 20-24. 